\title{
Export of functional Streptomyces coelicolor alditol oxidase to the periplasm or cell surface of Escherichia coli and its application in whole-cell biocatalysis
}

\author{
Edwin van Bloois • Remko T. Winter • Dick B. Janssen • \\ Marco W. Fraaije
}

Received: 15 December 2008 /Revised: 30 January 2009 / Accepted: 30 January 2009/Published online: 18 February 2009

(C) The Author(s) 2009. This article is published with open access at Springerlink.com

\begin{abstract}
Streptomyces coelicolor A3(2) alditol oxidase (AldO) is a soluble monomeric flavoprotein in which the flavin cofactor is covalently linked to the polypeptide chain. AldO displays high reactivity towards different polyols such as xylitol and sorbitol. These characteristics make AldO industrially relevant, but full biotechnological exploitation of this enzyme is at present restricted by laborious and costly purification steps. To eliminate the need for enzyme purification, this study describes a wholecell AldO biocatalyst system. To this end, we have directed AldO to the periplasm or cell surface of Escherichia coli. For periplasmic export, AldO was fused to endogenous $E$. coli signal sequences known to direct their passenger proteins into the $\mathrm{SecB}$, signal recognition particle (SRP), or Twin-arginine translocation (Tat) pathway. In addition, AldO was fused to an ice nucleation protein (INP)-based anchoring motif for surface display. The results show that Tat-exported AldO and INP-surface-displayed AldO are active. The Tat-based system was successfully employed in converting xylitol by whole cells, whereas the use of the INPbased system was most likely restricted by lipopolysaccharide LPS in wild-type cells. It is anticipated that these whole-cell systems will be a valuable tool for further biological and industrial exploitation of $\mathrm{AldO}$ and other cofactor-containing enzymes.
\end{abstract}

Keywords Carbohydrate oxidase . Whole cell biocatalysis · Flavo enzyme $\cdot$ Periplasmic transport $\cdot$ Surface display

E. van Bloois $\cdot$ R. T. Winter $\cdot$ D. B. Janssen $\cdot$ M. W. Fraaije $(\triangle)$ Laboratory of Biochemistry,

Groningen Biomolecular Sciences and Biotechnology Institute, University of Groningen,

Nijenborgh 4,

9747 AG Groningen, The Netherlands

e-mail: m.w.fraaije@rug.nl

\section{Introduction}

Carbohydrate oxidases oxidize their substrates with excellent regio- and/or enantioselectivity, which makes these enzymes of considerable industrial value. This is nicely illustrated by their use in diagnostics (biosensors for blood sugar) and in the food and drink industry (sweeteners and flavors; van Hellemond et al. 2006). Most carbohydrate oxidases belong to a distinct subgroup of sequence-related flavoproteins, namely the vanillyl-alcohol oxidase (VAO) family. Members of this family are characterized by a similar overall structure comprising a FAD binding domain and a substrate binding domain (Fraaije et al. 1998; Leferink et al. 2008). Recently, we identified a novel member of the VAO family in the proteome of Streptomyces coelicolor A3(2), called AldO (alditol oxidase). AldO is a soluble monomeric flavoprotein of $45.1 \mathrm{kDa}$ in which the flavin cofactor is covalently linked to the polypeptide chain (Heuts et al. 2007). The covalent anchoring of the FAD cofactor is an autocatalytic process and will only occur upon correct folding of the polypeptide chain. Therefore, the covalently incorporated FAD can be used as a folding reporter. AldO catalyzes the $\mathrm{C} 1$ oxidation of several polyols such as xylitol and sorbitol (Heuts et al. 2007). The recent determination of its atomic structure at a resolution of $1.1 \AA$ has revealed the structural features that determine the catalytic mechanism and substrate specificity of AldO (Forneris et al. 2008).

AldO is industrially relevant and can be used in various applications. At present, biotechnological exploitation of this enzyme is restricted by costly and laborious purification steps. It is therefore desirable to eliminate the need for enzyme purification using a whole-cell biocatalyst system. The use of AldO in such a system, however, is limited by the accessibility of substrates. Several strategies have been presented for Escherichia coli-based whole-cell biocatalyst 
systems to increase the accessibility of substrates. These include transport of foreign proteins to the periplasm and/or display at the cell surface (Samuelson et al. 2002; Mergulhao et al. 2005).

Proteins that function outside the cytoplasm (secretory proteins) can be exported to the periplasm of E. coli by different pathways. The vast majority of secretory proteins are exported across the inner membrane in an unfolded state via the Sec-translocon, which works as a protein conducting channel (Driessen and Nouwen 2008). Most secretory proteins are targeted to the Sec-translocon in a posttranslational fashion by the chaperone $\mathrm{SecB}$ in concert with the ATPase SecA, which drives protein translocation (Vrontou and Economou 2004; Zhou and $\mathrm{Xu} 2005$ ). A subset of secretory proteins is targeted to the Sec-translocon in a cotranslational fashion that involves the signal recognition particle (SRP) and its receptor FtsY (Luirink and Sinning 2004). In contrast to the Sec-translocon, the Twin-arginine translocation (Tat) system is able to export folded and often cofactor-containing secretory proteins to the periplasm posttranslationally (Lee et al. 2006).

Various systems have been described for display of foreign proteins on the surface of $E$. coli, including phage display and systems based on anchoring motifs derived from outer membrane proteins, lipoproteins, or auto-transporters (Samuelson et al. 2002; Lee et al. 2003). One such recently explored anchoring motif is derived from the ice-nucleation protein (INP) InaK from Pseudomonas syringae. This protein is attached to the cell surface by an glycosylphosphatidylinositol (GPI) anchor and comprises three structurally different domains: the non-repetitive $\mathrm{N}$ - and Cterminal domains and a repetitive central domain (Turner et al. 1991; Graether and Jia 2001). Both full-length INP and a truncated variant lacking the central domain are able to facilitate the surface display of foreign proteins, indicating that the non-repetitive domains are required for targeting and anchoring to the cell surface. Therefore, an INP derivative constituting the $\mathrm{N}$ - and $\mathrm{C}$-terminal domains is used to display foreign proteins on the surface of E. coli (Jung et al. 1998; Lee et al. 2000; Shimazu et al. 2001a; Cho et al. 2002; Kang et al. 2003; Li et al. 2004; Wu et al. 2006b; Yim et al. 2006).

In the present study, we have developed a whole-cell AldO biocatalyst system by directing AldO to the periplasm or cell surface of E. coli. For the transport across the inner membrane, we have explored several export systems. For periplasmic transport, AldO was fused to endogenous E. coli $\mathrm{Sec}$ and Tat signal sequences. A truncated INP derivative was used for surface display of AldO. The export of these constructs was analyzed by cellular fractionation and immunoblotting. Additionally, cellular fractions were assayed for oxidase activity. The results show that Tatexported AldO and INP-surface-displayed AldO are active. The Tat-based system was successfully employed in con- verting xylitol by whole cells, whereas the use of the INPbased system was most likely restricted by LPS in wild-type cells. It is anticipated that these whole-cell systems, cells decorated with the biocatalyst of interest, will be a valuable tool for further biological and industrial exploitation of AldO and other cofactor-containing enzymes.

\section{Materials and methods}

Reagents, enzymes, and sera

Restriction enzymes were from Roche Applied Science and New England Biolabs. Expand long template polymerase chain reaction $(\mathrm{PCR})$ system and $\mathrm{P} f u$ DNA polymerase were from Roche Applied Science and Invitrogen. Enhanced chemiluminescence (ECL) Western blotting detection reagent was from Amersham Biosciences. Horseradish peroxidase was from Fluka. All other chemicals were supplied by Sigma and of analytical grade. Antiserum against AldO was raised in a rabbit against the purified native protein and was prepared by Innovagen (Lund, Sweden). OmpA and DsbA antisera were kind gifts of H.D. Bernstein and J. Beckwith, respectively. DnaK antiserum was kindly provided by A. Mogk. Antiserum against riboflavin was from Abcam.

Strains, plasmids, and growth conditions

E. coli strains MC1061 (Casadaban and Cohen 1980) and Top10 (Invitrogen) were used as routine hosts for all plasmid constructs. Strains MC1061 and MC4100 (Casadaban 1976) were used for subcellular localization experiments and whole cell-based conversions of xylitol. The waaC null mutant JW3596-1 (GGSC no. 11805) has been described previously (Baba et al. 2006) and was obtained from the E. coli genetic stock center. JW3596-1 was used for the whole cell-based conversion of xylitol.

The plasmids pBAD-AldO and pBAD-MBP-AldO have been described earlier (Heuts et al. 2007). The different signal sequence chimeras were constructed as follows. For transport via the Tat pathway, the signal sequence of TorA and the first four amino acid residues of the mature protein were PCR-amplified from $E$. coli $\mathrm{K}-12$ genomic DNA. The PCR product was cloned NdeI/EcoRI into pBAD-MBPAldO, thereby replacing the N-terminal MBP tag and yielding pBAD-Tat-AldO. The plasmid pBAD-Sec-AldO, encoding an MalE signal sequence-AldO hybrid, was obtained in a similar fashion after PCR amplification of the MalE signal sequence using pMal-p2X (New England Biolabs) as template. SRP-AldO was obtained by PCR from pBAD-MBP-AldO as template and using a forward primer that included the codons for the DsbA signal 
sequence and the first two residues of the mature protein. The PCR product was cloned into pBAD (Invitrogen), yielding pBAD-SRP-AldO. For cell surface display of $\mathrm{AldO}$, a truncated variant of the inaK gene comprising the $\mathrm{N}-$ and $\mathrm{C}$-terminal domains was synthesized by Sloning BioTechnology (München, Germany) and subsequently cloned $N d e \mathrm{I} / E c o$ RI into vector $\mathrm{pBAD}-\mathrm{MBP}-\mathrm{AldO}$, thereby replacing the N-terminal MBP tag and yielding pBAD-INPAldO. Nucleotide sequences were verified by DNA sequencing. Primer sequences are available upon request.

Cultures were grown to saturation at $37^{\circ} \mathrm{C}$ overnight. The following day, overnight cultures were back-diluted $1: 100$ into fresh media containing $0.02 \%$ L-arabinose to induce the expression of $\mathrm{AldO}$ or its derivatives and grown for $48 \mathrm{~h}$ at $17^{\circ} \mathrm{C}$. All strains were routinely grown in LuriaBertani medium (per liter, $10 \mathrm{~g}$ tryptone, $5 \mathrm{~g}$ yeast extract, $5 \mathrm{~g} \mathrm{NaCl}$ ) under aerobic conditions unless indicated otherwise. Where appropriate, ampicillin $(100 \mu \mathrm{g} / \mathrm{ml})$ or kanamycin $(5 \mu \mathrm{g} / \mathrm{ml})$ were added to the culture medium.

\section{Cell fractionations}

Cells expressing AldO or its signal sequence derivatives were grown as described above. Fifteen to $20 \mathrm{OD}_{660}$ units of cells were harvested and fractionated into a spheroplast and periplasmic fraction as described (Huber et al. 2005). The cytoplasmic fraction was obtained as follows. After disruption of the spheroplasts by sonication and a brief clarifying spin, the clarified lysate was ultracentrifuged $\left(100,000 \times \mathrm{g}\right.$ for $40 \mathrm{~min}$ at $\left.4^{\circ} \mathrm{C}\right)$ and the supernatant was taken as the cytoplasmic fraction. Cells expressing INPAldO were grown as described above. Seventy-five $\mathrm{OD}_{660}$ units of cells were harvested and disrupted by sonication. The soluble, total membrane, inner membrane, and outer membrane fractions were obtained as described (Filip et al. 1973). In both fractionation procedures, proteins were precipitated by trichloroacetic acid and analyzed by sodium dodecyl sulfate-polyacrylamide gel electrophoresis (SDSPAGE) and immunoblotting.

\section{SDS-PAGE and immunoblotting}

Cellular fractions were normalized on the basis of the $\mathrm{OD}_{660}$, and samples of these fractions containing equal $\mathrm{OD}_{660}$ units were analyzed on standard $12 \%$ SDS-PAGE gels. Proteins were transferred to nitrocellulose membrane (Amersham Biosciences) using a semidry apparatus from Biorad. Immunodetection was performed using the primary antisera described above, a secondary horseradishperoxidase-coupled antiserum (Rockland), and the ECL system from Amersham Biosciences (according to the instructions of the manufacturer). Proteins were visualized using the Fujifilm LAS-3000 imaging system.
Analytical methods

The oxidase activity of cellular fractions and whole cells expressing Tat-AldO or INP-AldO was determined by coupling the production of $\mathrm{H}_{2} \mathrm{O}_{2}$ by $\mathrm{AldO}$ or its derivatives to a horseradish-peroxidase-mediated oxidation of 4aminoantipyrine and 3,5-di-chloro-2-hydroxybenzenesulfonic acid. The resulting pink adduct can be detected spectrophotometrically at $515 \mathrm{~nm}\left(\varepsilon_{515}=26 \mathrm{mM}^{-1} \mathrm{~cm}^{-1}\right.$; Federico et al. 1997). For the detection of oxidase activity, phosphate-buffered saline ( $\mathrm{pH}$ 7.4) was used as assay buffer containing $0.1 \mathrm{mM} 4$-aminoantipyrine, $1 \mathrm{mM} 3$,5-di-chloro2-hydroxybenzenesulfonic acid, $3 \mathrm{U}$ horseradish peroxidase, $5 \mathrm{mM}$ xylitol, $0.01 \mathrm{OD}_{660}$ units of cells (expressing Tat-AldO or INP-AldO) or samples of cellular fractions. Prior to the analysis of oxidase activity, total membrane fractions were detergent-solubilized ( $2 \% n$-dodecyl- $\beta$-Dmaltoside). Alkaline phosphatase activity was measured spectrophotometrically at $405 \mathrm{~nm}$ (Brickman and Beckwith 1975) using Tris-buffered saline (pH 7.4) as assay buffer containing $1.2 \mu \mathrm{g} / \mu \mathrm{l}$ phosphatase substrate (Sigma; $\varepsilon_{405}=$ $12.5 \mathrm{mM}^{-1} \mathrm{~cm}^{-1}$ ) and $0.02 \mathrm{OD}_{660}$ units of cells (expressing INP-AldO). All activity measurements were performed at ambient temperature, and where appropriate $5 \mathrm{mM} \mathrm{MgCl} 2$ was included in the assay buffer to stabilize the outer membrane.

\section{Results}

Design of fusion constructs and experimental strategy

Here, we have developed a whole-cell AldO biocatalyst system by directing $S$. coelicolor AldO to the periplasm or cell surface of $E$. coli. For periplasmic transport, we have fused AldO N-terminally to the signal sequences of the endogenous E. coli proteins MalE, DsbA, or TorA, known to direct their substrates into the SecB, SRP, or Tat pathway, respectively (Kumamoto and Beckwith 1985; Schierle et al. 2003; Tullman-Ercek et al. 2007). Additionally, we have used a truncated INP variant comprising the InaK N- and $\mathrm{C}$-terminal domain and lacking the central repeating domain for surface display. This INP derivative was fused N-terminally to AldO (Table 1). All fusions are based on a cassette system (Fig. 1) in which the coding region of aldo is cloned EcoRI/HindIII downstream of the indicated export signal, giving rise to Sec-AldO, SRP-AldO, Tat-Aldo, and INP-AldO. Wild-type AldO, lacking a signal sequence, is included in the present study as a negative control. Expression of all constructs is under control of the arabinose-inducible $\mathrm{P}_{\mathrm{BAD}}$ promotor, allowing moderate overexpression to prevent saturation of export pathways and accompanying cell toxicity. 
Table 1 Sequences of export signals used in this study

\begin{tabular}{ll}
\hline Signal peptide & Sequence $^{\text {a }}$ \\
\hline MalE & MKIKTGARILALSALTTMMFSASALA \\
DsbA & MKKIWLALAGLVLAFSASAAQ \\
TorA & MNNNDLFQASRRRFLAQLGGLTVAGMLGPSLLTPRRATAAQAA \\
INP $\left(\right.$ InaK $\left._{\mathrm{NC}}\right)$ & MTLDKALVLRTCANNMADHCGLIWPASGTVESRYWQSTRRHENGLVGLLWGAGTSAFLSVHADARWIV \\
& CEVAVADIISLEEPGMVKFPRAEVVHVGDRISASHFISARQADPASTSTSTSTSTLTPMPTAIPTPMPAVASV \\
& TLPVAEQARHEVFDVASVSAAAAPVNTLPVTTPQNLQTATYGSTLSGDNHSRLIAGYGSNETAGNHSDLI \\
& GGHDCTLMAGDQSRLTAGKNSVLTAGARSKLIGSEGSTLSAGEDSTLIFRLWDGKRYRQLVARTGENGV \\
& EADIPYYVNEDDDIVDKPDEDDDWIEVK
\end{tabular}

${ }^{\mathrm{a}}$ The sequence is given in the $\mathrm{N}$ - to $\mathrm{C}$-terminal direction

Previous work indicated that AldO expressed in E. coli is a soluble protein in agreement with a cytoplasmic localization as predicted by PSORT (Nakai and Horton 1999; Heuts et al. 2007). To unambiguously assess its cellular localization, we first studied the localization of wild-type AldO in E. coli. Therefore, cells expressing wildtype $\mathrm{AldO}$ were grown to saturation at $17^{\circ} \mathrm{C}$, harvested,
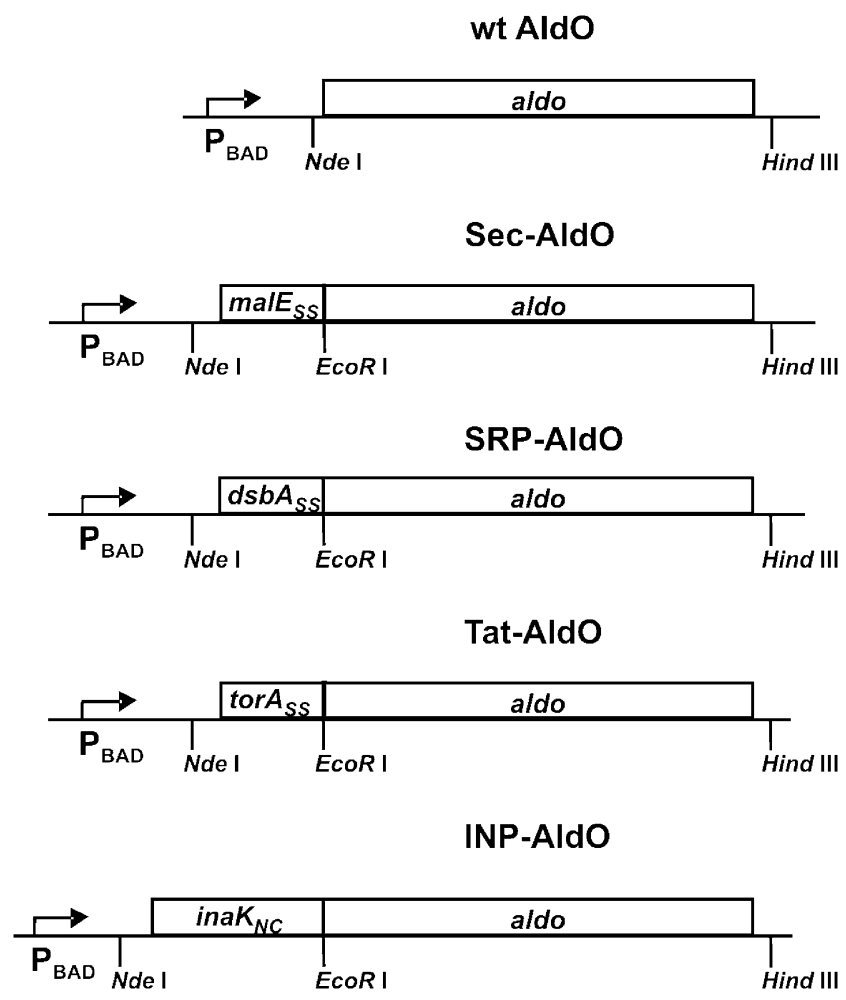

Fig. 1 Schematic representation of the hybrid constructs used. All fusions were constructed as described in "Materials and methods". For Sec-dependent export, AldO was fused to the signal sequences ( $s s$ ) of MalE or DsbA, resulting in Sec-AldO or SRP-AldO, respectively. For export by the Tat-system, AldO was fused to the signal sequence of TorA, yielding Tat-AldO. AldO was fused to a truncated variant of INP comprising the $\mathrm{N}$ - and C-terminal domains for surface display. This construct was termed INP-AldO. Expression of all constructs is driven from an arabinose-inducible $\mathrm{P}_{\mathrm{BAD}}$ promotor and relevant restriction sites are indicated converted to spheroplasts by EDTA/lysozyme treatment, after which the periplasmic fraction was obtained by osmotic shock. Subsequently, the spheroplasts were disrupted by sonication and subjected to ultracentrifugation to obtain the cytoplasmic fraction. The different subcellular fractions were analyzed by immunoblotting using antisera directed against AldO or riboflavin to study (1) the AldO content of the samples and (2) whether AldO contained covalently bound FAD. Figure 2a shows that wild-type AldO is cytoplasmically localized and contains covalently bound FAD as previously reported (Heuts et al. 2007). This indicates that AldO is properly folded, as evidenced by the presence of covalent FAD, and functional, as confirmed by the detection of oxidase activity in the cytoplasmic fraction.

As controls to monitor the efficiency of the fractionation procedure, the levels of DnaK and DsbA, which serve as cytoplasmic or periplasmic marker, were analyzed in the same samples by immunoblotting. The data show that DnaK is restricted to the cytoplasmic fraction and DsbA is mainly detected in the periplasmic fraction, demonstrating the efficiency of the fractionation procedure and thereby validating our assay conditions.

Sec-dependent export of AldO to the periplasm

To examine whether AldO could be functionally transported to the periplasm via the Sec-translocon, $\mathrm{AldO}$ was fused to the MalE (Sec-AldO) or DsbA (SRP-AldO) signal sequence.

First, we studied the export of Sec-AldO by cell fractionation and immunoblotting (Fig. 2b). The results show that Sec-AldO is exported to the periplasm. However, Sec-AldO does not contain covalently bound FAD, unlike purified wild-type AldO, which was included as a positive control (indicated by an arrowhead) in this immunoblot experiment. The absence of FAD indicates that Sec-exported AldO is not folded into its biologically active conformation which was verified by the lack of oxidase activity in the cytoplasmic and periplasmic fraction. Notably, the inability to covalently incorporate FAD destabilizes AldO, resulting in degradation and cytoplasmic aggregation of AldO as indicated by its co- 
Fig. 2 Subcellular localization of AldO of wild-type AldO (a), Sec-AldO (b), SRP-AldO (c), and Tat-AldO (d). E. coli cells expressing the indicated constructs were fractionated into total cells $(T)$, cytoplasm $(C)$, and periplasm $(P)$. Samples were normalized on the basis of $\mathrm{OD}_{660}$ and analyzed by immunoblotting with the indicated antisera. Black lines indicate that intervening lanes have been spliced out. Oxidase activity in the cytoplasmic and periplasmic fraction was determined using xylitol as substrate. Plus sign, oxidase activity; minus sign, no oxidase activity a
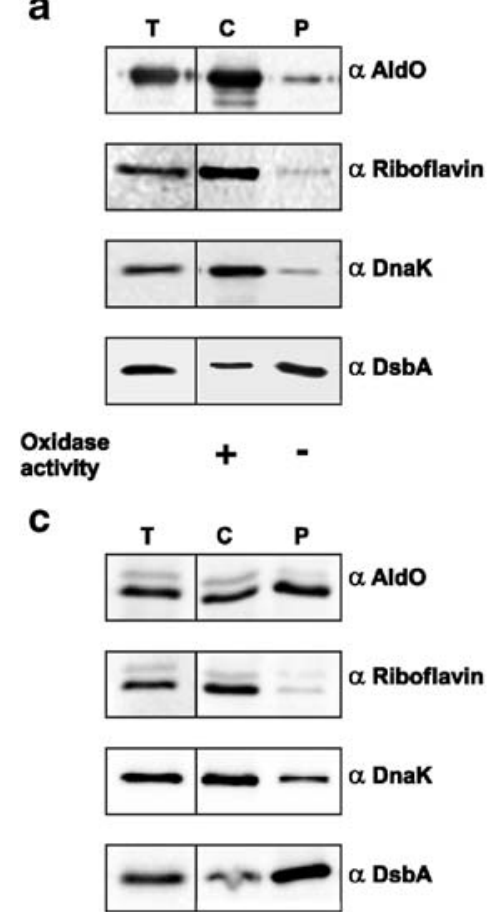

b
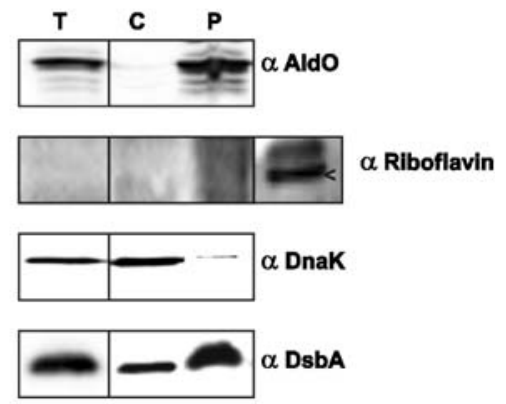

Oxidase activity

d
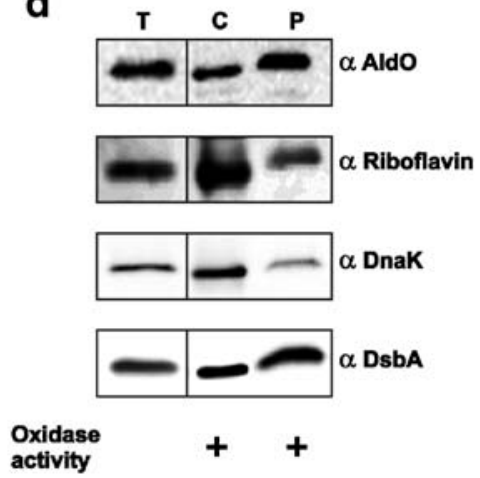

fractionation with other insoluble proteins (data not shown). The efficiency of the fractionation procedure was confirmed by analyzing the DnaK and DsbA levels in the same samples by immunoblotting.

Next, we investigated the periplasmic export of SRP-AldO using the same experimental setup as described above. Figure 2c shows that SRP-AldO is transported to the periplasm similar to Sec-AldO. Remarkably, a considerable amount of mature-sized AldO is present in the cytoplasmic fraction, which may result from degradation of the DsbA signal sequence by cytoplasmic proteases. This cytoplasmic population of mature-sized AldO contains covalently bound FAD, indicating that it is folded into its active conformation and displays oxidase activity as expected. In contrast, periplasmic AldO does not contain FAD and is not active. Control blots were performed using antisera against DnaK and DsbA, showing that the fractionation protocol is effective.

The results above show that Sec-exported AldO is not active because FAD is not assembled properly into the protein. Apparently, cofactor incorporation is blocked due the inherent requirement of the protein to be unfolded for Sec-dependent export (Driessen and Nouwen 2008).

Tat-dependent export of AldO to the periplasm

In contrast to the Sec-translocon, the Tat-system is able to transport folded and often cofactor containing proteins to the periplasm (Lee et al. 2006). To test whether this pathway can be used for transport of active AldO to the periplasm, we fused AldO to the Tat signal sequence of TorA (Tat-AldO). The export of Tat-AldO was assayed by cell fractionation and immunoblotting. As shown in Fig. 2d, a substantial proportion of AldO is clearly exported to the periplasm, whereas a considerable amount of mature-sized $\mathrm{AldO}$ is also present in the cytoplasmic fraction, which may result from cytoplasmic degradation of the TorA signal sequence as observed previously with a GFP-TorA signal sequence chimera and similar to SRP-AldO (Fig. 2c; Thomas et al. 2001). Interestingly, the cytoplasmic and periplasmic populations of mature-sized AldO contain covalently linked FAD, indicating that AldO is folded into its active conformation as confirmed by the detection of oxidase activity in both fractions. The efficiency of the fractionation procedure was verified by analyzing the DnaK and DsbA content in the same samples by immunoblotting.

In conclusion, these data show that AldO can be transported to the periplasm in an active form by the Tat pathway.

Surface display of AldO

AldO was displayed on the surface of E. coli using a truncated INP variant. To examine outer membrane localization of INP-AldO, cells expressing this construct were fractionated into a soluble (cytoplasm and periplasm) and total membrane fraction. The total membrane fraction 
was further separated into an inner membrane and outer membrane fraction by sarcosyl extraction (Filip et al. 1973). Samples were analyzed by immunoblotting using the indicated antisera. Figure $3 \mathrm{a}$ shows that INP-AldO is detected in the cell extract, soluble fraction, and total membrane fraction. After sarcosyl extraction, INP-AldO is exclusively observed in the outer membrane fraction, suggesting that INP-AldO is indeed localized to the outer membrane. This is in agreement with other recent studies using INP for the surface display of foreign proteins in $E$. coli (Lee et al. 2000; Li et al. 2004; Wu et al. 2006b). Moreover, INP-AldO present in these fractions clearly contains covalently bound FAD. This indicates that INPAldO is properly folded and functional as confirmed by the detection of oxidase activity in the cell extract, soluble fraction, and total membrane fraction. Unfortunately, no oxidase activity could be detected in the inner membrane and outer membrane fractions after sarcosyl extraction, probably due to inactivation of AldO by the detergent used.

As controls to verify the efficiency of the fractionation protocol, OmpA and DsbA levels in the same samples were analyzed by immunoblotting. OmpA, which serves as an outer membrane marker, is, as expected, mainly detected in the outer membrane fraction together with INP-AldO. DsbA, which serves as a soluble marker, is exclusively detected in the soluble fraction.

Thus far, the data show that a considerable fraction of FAD-containing INP-AldO is present in outer membrane. However, these data do not confirm whether this protein is a

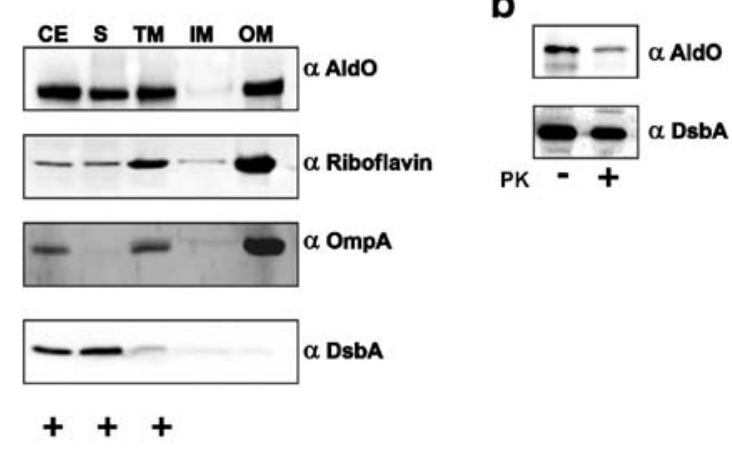

Fig. 3 Subcellular localization of INP-AldO (a). E. coli cells expressing INP-AldO were fractionated into a cell extract $(C E)$, soluble $(S)$, and total membrane $(T M)$ fraction. The total membrane fraction was further separated into an inner membrane $(I M)$ and outer membrane $(O M)$ fraction by extraction with $0.5 \%$ sarcosyl. Samples were normalized on the basis of $\mathrm{OD}_{660}$ and analyzed by immunoblotting with the indicated antisera. Oxidase activity in the cell extract, soluble, and total membrane fraction was determined using xylitol as substrate. Plus sign, oxidase activity; minus sign, no oxidase activity. b Protease accessibility analysis of surface displayed INP-AldO. E. coli cells expressing INP-AldO were treated (plus sign) or mocktreated (minus sign) with proteinase $\mathrm{K}(P K)$ to degrade cell surface proteins. Samples were normalized on the basis of $\mathrm{OD}_{660}$ and analyzed by immunoblotting with the indicated antisera indeed surface-localized. To verify surface localization, the accessibility of INP-AldO towards exogenously added proteinase $\mathrm{K}$ on whole cells was tested. Samples were analyzed by immunoblotting using the indicated antisera (Fig. 3b). Clearly, INP-AldO is almost completely degraded after proteinase $\mathrm{K}$ treatment when compared to mocktreated cells. To monitor the integrity of the cells, we analyzed the levels of the periplasmic protein DsbA in the same samples which were unaltered by proteinase $\mathrm{K}$ treatment. These data confirm that INP-AldO is indeed surface-localized.

Combined, the results show that AldO is successfully displayed at the surface of $E$. coli using a truncated INP variant. Notably, AldO presented at the cell surface contains covalently bound FAD, suggesting that it has attained a correctly folded and active conformation.

Periplasmic localized AldO and surface-displayed AldO as whole-cell biocatalysts

The export of active AldO to the periplasm or cell surface is of considerable value as it may facilitate the use of these systems in whole-cell biocatalysis. As proof of principle, we therefore assayed spectrophotometrically whether wildtype E. coli cells expressing AldO, Tat-AldO, or INP-AldO could be successfully employed in the conversion of xylitol. A representative data set is shown in Fig. 4. As expected, control cells expressing wild-type AldO did not show significant conversion of xylitol. This suggests that no substantial lysis of the cells has occurred under these conditions. Only upon permeabilization of the cells with toluene was significant conversion of xylitol observed, indicating that cytoplasmic localized, wild-type AldO is unable to react with xylitol probably because xylitol is unable to pass the inner membrane. In the absence of arabinose, no conversion is observed. Interestingly, xylitol is readily converted by cells expressing Tat-AldO. Permeabilization of these cells did not further improve oxidase activity. In contrast, cells expressing INP-AldO only displayed oxidase activity upon permeabilization by toluene, suggesting that the activity and/or substrate accessibility of INP-AldO is compromised in wild-type cells.

From these data, we conclude that export of AldO to the periplasm by the Tat-system is a successful strategy for applying this protein in whole-cell biocatalysis. Cellsurface-displayed AldO, however, showed an unanticipated poor activity with xylitol. It is conceivable that prominent endogenous surface molecules, such as lipopolysaccharides (LPS), inhibit INP-AldO and/or restrict the accessibility of xylitol. To test whether AldO displayed on cells containing truncated LPS molecules is able to convert xylitol successfully, we used the E. coli waaC null strain JW3596-1 (Baba et al. 2006). The waaC gene product, a heptosyltransferase, 
Fig. 4 Conversion of xylitol by E. coli cells expressing TatAldO or INP-AldO. E. coli cells were grown in the absence or presence of arabinose to induce the expression of the indicated constructs; cells were harvested and resuspended in PBS. The conversion of xylitol, using equal amounts of cells, was monitored spectrophotometrically at room temperature. As a negative control, cells expressing wild-type (wt) AldO were included. Cells were permeabilized by the addition of $2 \%$ toluene, as indicated. The conversion of xylitol is given as the relative whole-cell activity normalized on the basis of $\mathrm{OD}_{660}$

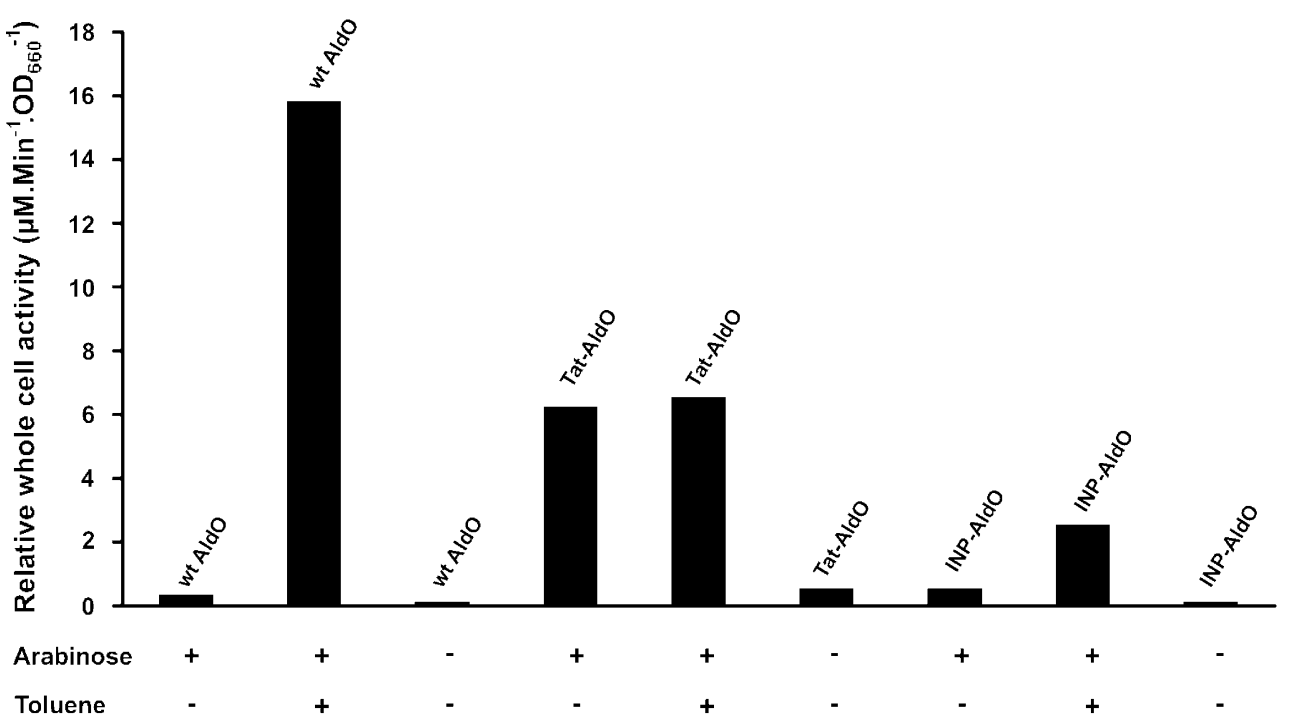

biocatalyst systems, we directed AldO to the periplasm or cell surface of E. coli. For Sec-dependent export to the periplasm, we have fused AldO N-terminally to the endogenous $E$. coli signal sequences of MalE or DsbA. The MalE signal sequence, which directs its substrate into the posttranslational SecB pathway, is commonly used for the periplasmic transport of recombinant proteins (Kumamoto and Beckwith 1985; Mergulhao et al. 2005). Although the DsbA signal sequence is less frequently used for this purpose, several studies have reported its successful application in directing passenger proteins to the periplasm via the co-translational SRP pathway (Schierle et al. 2003; Huber et al. 2005; Marrichi et al. 2008). Additionally, we have fused AldO N-terminally to the signal sequence of TorA for Tatdependent export to the periplasm. The TorA signal sequence is considered to be highly Tat-specific and is commonly employed for the transport of heterologous proteins to the periplasm (Cristobal et al. 1999; Bruser 2007; TullmanErcek et al. 2007). The export of these fusion constructs was examined by cellular fractionation and immunoblotting. The data show that all fusion constructs are directed to the periplasm. However, only Tat-exported AldO was fully active as shown by proper cofactor assembly and oxidase activity in the periplasmic fraction. Similarly, GFP, which is only able to fold in the cytoplasm, was transported to the $E$. coli periplasm when fused to a Sec or Tat signal sequence, but only Tat-exported GFP was active (Feilmeier et al. 2000; Thomas et al. 2001). This result is explained by the export of proteins in a pre-folded, active conformation by the Tat system in contrast to Sec-dependent translocation (Lee et al. 2006). Therefore, our data reinforce the notion that the Tat system is dedicated to the export of pre-folded and often cofactor containing proteins to the periplasm.

Different systems have been described for display of foreign proteins on the cell surface of bacteria. For E. coli, which often constitutes a bottleneck in developing whole-cell 
these include phage display and systems based on surface anchoring motifs derived of outer membrane proteins, lipoproteins, and auto-transporters (Graether and Jia 2001; Samuelson et al. 2002; Lee et al. 2003; Jose and Meyer 2007). Most of these systems rely on the Sec-translocon for initial transport across the inner membrane. This requires the protein to be unfolded, which interferes with cofactor assembly and activity of AldO as indicated by our data, and therefore, these systems are ruled out for proper export. An alternative system based on an anchoring motif from the ice nucleation protein InaK from $P$. syringae is commonly used for surface display of foreign proteins in $E$. coli, such as viral antigens, single chain antibodies, and enzymes (Jung et al. 1998; Lee et al. 2000; Shimazu et al. 2001a; Cho et al. 2002; Kang et al. 2003; Li et al. 2004; Wu et al. 2006b; Yim et al. 2006). The structural diversity of these surfacedisplayed proteins emphasizes the remarkable tolerance and flexibility of the INP system. INP is bound to the cell surface by a GPI anchor and comprises three structurally different domains: a non-repetitive $\mathrm{N}$ - and C-terminal domain and a central repetitive domain (Turner et al. 1991; Graether and Jia 2001). The molecular mechanism by which INP facilitates surface display is not clear as no signal sequence could be identified (Schmid et al. 1997). In this respect, it is interesting to note that our data indicate that the export mechanism of INP most likely does not involve the Sec-translocon for translocation across the inner membrane, as surface-displayed INP-AldO contains covalently bound FAD and is functional in contrast to Sec-AldO and SRP-AldO. Different studies suggest that the nonrepetitive domains are required for targeting and anchoring to the cell surface (Lee et al. 2000; Shimazu et al. 2001a, b; $\mathrm{Wu}$ et al. 2006b). However, recent evidence indicates that the N-terminal domain alone is sufficient for cell surface display ( $\mathrm{Li}$ et al. 2004; Wu et al. 2006a). In the present work, we have used a truncated version of INP comprising the InaK N- and C-terminal domains for surface display of AldO in E. coli. The cellular localization of this construct was analyzed by cellular fractionation and immunoblotting. The data show that INP-AldO is active as judged by the proper FAD incorporation and oxidase activity in the cell extract, soluble fraction, and total membrane fraction. As expected, INP-Aldo is localized to the outer membrane and is surface-localized as demonstrated by its accessibility towards exogenously added proteinase $\mathrm{K}$.

Compartmentalization of cells is employed by nature to create catalytically efficient environments as exemplified by the periplasmic or extracellular localization of enzymes in E. coli, which may be beneficial in cases where, for example, the substrate/product of interest cannot cross the membrane or where the substrate/product is also converted by another intracellular enzyme. Our data illustrate this by showing that wild-type E. coli cells expressing Tat-AldO are able to convert xylitol, whereas cells expressing wildtype AldO cannot. Surprisingly, cells expressing surfacedisplayed INP-AldO are unable to react with xylitol. Conceivably, INP-AldO may be inhibited and/or the accessibility of xylitol may be restricted in wild-type cells by a prominent cell surface molecule, such as LPS. Indeed, surface-displayed AldO on cells of an E. coli mutant containing truncated LPS molecules was able to convert xylitol successfully, consistent with the idea that in wildtype cells, LPS restricts the availability of cell surfacedisplayed AldO for whole-cell biocatalysis.

These data nevertheless demonstrate proof of principle and will further the exploitation of AldO in broad biological and industrial applications. Furthermore, the strategies presented for periplasmic export and surface display are also applicable to other enzymes and, in particular, cofactor-containing enzymes.

Acknowledgment This research is supported by the Dutch Technology Foundation STW, Applied Science Division of NWO, and the Technology Program of the Ministry of Economic Affairs.

Open Access This article is distributed under the terms of the Creative Commons Attribution Noncommercial License which permits any noncommercial use, distribution, and reproduction in any medium, provided the original author(s) and source are credited.

\section{References}

Baba T, Ara T, Hasegawa M, Takai Y, Okumura Y, Baba M, Datsenko KA, Tomita M, Wanner BL, Mori H (2006) Construction of Escherichia coli K-12 in-frame, single-gene knockout mutants: the Keio collection. Mol Syst Biol 2:2006-0008

Brickman E, Beckwith J (1975) Analysis of the regulation of Escherichia coli alkaline phosphatase synthesis using deletions and phi80 transducing phages. J Mol Biol 96:307-316

Bruser T (2007) The twin-arginine translocation system and its capability for protein secretion in biotechnological protein production. Appl Microbiol Biotechnol 76:35-45

Casadaban MJ (1976) Transposition and fusion of the lac genes to selected promoters in Escherichia coli using bacteriophage lambda and mu. J Mol Biol 104:541-555

Casadaban MJ, Cohen SN (1980) Analysis of gene control signals by DNA fusion and cloning in Escherichia coli. J Mol Biol 138:179-207

Cho CM, Mulchandani A, Chen W (2002) Bacterial cell surface display of organophosphorus hydrolase for selective screening of improved hydrolysis of organophosphate nerve agents. Appl Environ Microbiol 68:2026-2030

Cristobal S, de Gier JW, Nielsen H, von Heijne G (1999) Competition between Sec- and TAT-dependent protein translocation in Escherichia coli. EMBO J 18:2982-2990

Driessen AJ, Nouwen N (2008) Protein translocation across the bacterial cytoplasmic membrane. Annu Rev Biochem 77:643667

Federico R, Angelini R, Ercolini L, Venturini G, Mattevi A, Ascenzi P (1997) Competitive inhibition of swine kidney copper amine 
oxidase by drugs: amiloride, clonidine, and gabexate mesylate. Biochem Biophys Res Commun 240:150-152

Feilmeier BJ, Iseminger G, Schroeder D, Webber H, Phillips GJ (2000) Green fluorescent protein functions as a reporter for protein localization in Escherichia coli. J Bacteriol 182:40684076

Filip C, Fletcher G, Wulff JL, Earhart CF (1973) Solubilization of the cytoplasmic membrane of Escherichia coli by the ionic detergent sodium-lauryl sarcosinate. J Bacteriol 115:717-722

Forneris F, Heuts DP, Delvecchio M, Rovida S, Fraaije MW, Mattevi A (2008) Structural analysis of the catalytic mechanism and stereoselectivity in Streptomyces coelicolor alditol oxidase. Biochemistry 47:978-985

Fraaije MW, Van Berkel WJ, Benen JA, Visser J, Mattevi A (1998) A novel oxidoreductase family sharing a conserved FAD-binding domain. Trends Biochem Sci 23:206-207

Frirdich E, Whitfield C (2005) Lipopolysaccharide inner core oligosaccharide structure and outer membrane stability in human pathogens belonging to the Enterobacteriaceae. J Endotoxin Res 11:133-144

Graether SP, Jia Z (2001) Modeling Pseudomonas syringae icenucleation protein as a beta-helical protein. Biophys J 80:11691173

Heuts DP, van Hellemond EW, Janssen DB, Fraaije MW (2007) Discovery, characterization, and kinetic analysis of an alditol oxidase from Streptomyces coelicolor. J Biol Chem 282:2028320291

Huber D, Boyd D, Xia Y, Olma MH, Gerstein M, Beckwith J (2005) Use of thioredoxin as a reporter to identify a subset of Escherichia coli signal sequences that promote signal recognition particle-dependent translocation. J Bacteriol 187:2983-2991

Jose J, Meyer TF (2007) The autodisplay story, from discovery to biotechnical and biomedical applications. Microbiol Mol Biol Rev 71:600-619

Jung HC, Lebeault JM, Pan JG (1998) Surface display of Zymomonas mobilis levansucrase by using the ice-nucleation protein of Pseudomonas syringae. Nat Biotechnol 16:576-580

Kang SM, Rhee JK, Kim EJ, Han KH, Oh JW (2003) Bacterial cell surface display for epitope mapping of hepatitis $\mathrm{C}$ virus core antigen. FEMS Microbiol Lett 226:347-353

Kumamoto CA, Beckwith J (1985) Evidence for specificity at an early step in protein export in Escherichia coli. J Bacteriol 163:267274

Lee JS, Shin KS, Pan JG, Kim CJ (2000) Surface-displayed viral antigens on Salmonella carrier vaccine. Nat Biotechnol 18:645648

Lee SY, Choi JH, Xu Z (2003) Microbial cell-surface display. Trends Biotechnol 21:45-52

Lee PA, Tullman-Ercek D, Georgiou G (2006) The bacterial twinarginine translocation pathway. Annu Rev Microbiol 60:373-395

Leferink NG, Heuts DP, Fraaije MW, van Berkel WJ (2008) The growing VAO flavoprotein family. Arch Biochem Biophys 474:292-301

Li L, Kang DG, Cha HJ (2004) Functional display of foreign protein on surface of Escherichia coli using N-terminal domain of ice nucleation protein. Biotechnol Bioeng 85:214-221

Luirink J, Sinning I (2004) SRP-mediated protein targeting: structure and function revisited. Biochim Biophys Acta 1694:17-35
Marrichi M, Camacho L, Russell DG, DeLisa MP (2008) Genetic toggling of alkaline phosphatase folding reveals signal peptides for all major modes of transport across the inner membrane of bacteria. J Biol Chem 283:35223-35235

Mergulhao FJ, Summers DK, Monteiro GA (2005) Recombinant protein secretion in Escherichia coli. Biotechnol Adv 23:177202

Nakai K, Horton P (1999) PSORT: a program for detecting sorting signals in proteins and predicting their subcellular localization. Trends Biochem Sci 24:34-36

Raetz CR, Whitfield C (2002) Lipopolysaccharide endotoxins. Annu Rev Biochem 71:635-700

Samuelson P, Gunneriusson E, Nygren PA, Stahl S (2002) Display of proteins on bacteria. J Biotechnol 96:129-154

Schierle CF, Berkmen M, Huber D, Kumamoto C, Boyd D, Beckwith J (2003) The DsbA signal sequence directs efficient, cotranslational export of passenger proteins to the Escherichia coli periplasm via the signal recognition particle pathway. J Bacteriol 185:5706-5713

Schmid D, Pridmore D, Capitani G, Battistutta R, Neeser JR, Jann A (1997) Molecular organisation of the ice nucleation protein InaV from Pseudomonas syringae. FEBS Lett 414:590-594

Shimazu M, Mulchandani A, Chen W (2001a) Cell surface display of organophosphorus hydrolase using ice nucleation protein. Biotechnol Prog 17:76-80

Shimazu M, Mulchandani A, Chen W (2001b) Simultaneous degradation of organophosphorus pesticides and $p$-nitrophenol by a genetically engineered Moraxella sp. with surface-expressed organophosphorus hydrolase. Biotechnol Bioeng 76:318-324

Thomas JD, Daniel RA, Errington J, Robinson C (2001) Export of active green fluorescent protein to the periplasm by the twinarginine translocase (Tat) pathway in Escherichia coli. Mol Microbiol 39:47-53

Tullman-Ercek D et al (2007) Export pathway selectivity of Escherichia coli twin arginine translocation signal peptides. J Biol Chem 282:8309-8316

Turner MA, Arellano F, Kozloff LM (1991) Components of ice nucleation structures of bacteria. J Bacteriol 173:6515-6527

van Hellemond EW, Leferink NG, Heuts DP, Fraaije MW, van Berkel WJ (2006) Occurrence and biocatalytic potential of carbohydrate oxidases. Adv Appl Microbiol 60:17-54

Vrontou E, Economou A (2004) Structure and function of SecA, the preprotein translocase nanomotor. Biochim Biophys Acta 1694:67-80

Wu ML, Tsai CY, Chen TH (2006a) Cell surface display of Chi92 on Escherichia coli using ice nucleation protein for improved catalytic and antifungal activity. FEMS Microbiol Lett 256:119-125

Wu PH, Giridhar R, Wu WT (2006b) Surface display of transglucosidase on Escherichia coli by using the ice nucleation protein of Xanthomonas campestris and its application in glucosylation of hydroquinone. Biotechnol Bioeng 95:1138-1147

Yim SK, Jung HC, Pan JG, Kang HS, Ahn T, Yun CH (2006) Functional expression of mammalian NADPH-cytochrome P450 oxidoreductase on the cell surface of Escherichia coli. Protein Expr Purif 49:292-298

Zhou J, Xu Z (2005) The structural view of bacterial translocationspecific chaperone SecB: implications for function. Mol Microbiol 58:349-357 